Rev. Elev. Méd. vét. Pays trop., 1973, 26 (3) : 349-62

\title{
La graine de coton en embouche intensive. Performances comparées des zébus, des taurins et des produits de leur croisement.
}

\author{
H. CALvet $(*)$, J. VAlenZA $\left({ }^{* *}\right)$, D. FRIOT $(*)$, A. M. WANE $\left({ }^{* *}\right)$
}

\begin{abstract}
RESUME
L'expérience rapportée dans cette note a pour but de tester et comparer les réponses à l'embouche intensive des quatre races de bovins zébus Maure et Gobra, taurin Ndama et métis naturel Djakoré que l'on rencontre au Sénégal. L'aliment utilisé, à base de coque d'arachide mélassée, de graine de coton et de farine basse de riz est distribué à volonté pendant six semaines, puis rationné. Les animaux sont des taurillons de 4 à 5 ans, d'un poids moyen de 200 à $245 \mathrm{~kg}$.

Les résultats enregistrés sont les suivants :

- croît moyen quotidien de 809 à $1.152 \mathrm{~g}$ selon les lots,

- indice moyen de consommation de 8,88 à 6,67 ,

mettant en évidence la bonne réponse de ces animaux à l'embouche avec un net avantage pour les zébus et en particulier pour les Maures.

Ce type de ration se révèle être d'une bonne rentabilité surtout chez les zébus et se situe parmi les meilleurs des points de vue performance et coût de production du $\mathrm{kg}$ de croît.
\end{abstract}

Un deuxième est de comparer les performances de différents types d'animaux, utilisables au Sénégal en embouche intensive. Quatre lots ont ainsi été constitués, comprenant des zébus (Maures et Gobra), des taurins de race Ndama et des animaux résultant du croisement des deux espèces, nombreux au Sénégal où ils sont connu sous le vocable de «Djakoré ».

\section{MATERIEL ET METHODES}

Le protocole utilisé est comparable à celui des essais antérieurs. L'expérimentation se déroule à Sangalkam et utilise les parcs d'embouche aménagés depuis plusieurs années.

(*) Laboratoire National de l'Elevage, B.P. 2057, Dakar-Hann, Sénégal.

(**) Laboratoire National de l'Elevage, Sangalkam, Sénégal.
Tous les animaux mis en expérience sont des mâles entiers âgés de 3 à 5 ans, dont le poids au début est compris entre 225 et $245 \mathrm{~kg}$.

Le lot 1 comprend 10 taurillons zébus Maures.

Le lot 2 comprend 10 taurillons zébus Gobra.

Le lot 3 comprend 10 métis Djakoré.

Le lot 4 comprend 10 taurins de race Ndama.

\section{La ration}

L'élément de base de la ration se compose de 36 p. 100 de coque mélassée. La proportion de graine de coton a dû changer en cours d'essai, de même que la nature $d_{2}$, farines utilisées. 
TABLEAU N $\mathrm{N}^{\circ}$ I

Composition des rations

\begin{tabular}{|c|c|c|c|c|}
\hline Composition & $\begin{array}{l}\text { Aliment I } \\
30.4 .72\end{array}$ & $\begin{array}{c}\text { Aliment II } \\
26.5 .72\end{array}$ & $\begin{array}{l}\text { Aliment III } \\
23.6 .72\end{array}$ & $\begin{array}{c}\text { Aliment IV } \\
18.8 .72\end{array}$ \\
\hline Coque d'arachide & 20 & 29 & 20 & 20 \\
\hline Mêlasse & 16 & 15 & 1.6 & 16 \\
\hline Graine de coton & 27 & 18 & 24 & 24 \\
\hline Son de maïs & 20 & 10 & 21,5 & 21,5 \\
\hline Farine de riz & 14 & 20 & 14 & - \\
\hline Sorgho & 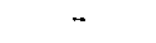 & - & - & 14 \\
\hline Tourteau d'axachide & - & 3 & 1 & 1 \\
\hline Urêe & 1 & 1 & 1 & 1 \\
\hline Phosphate bicalcique & - & 1,5 & 0,5 & 0,5 \\
\hline Carbonate de chaux & 1 & 1 & 1 & 1 \\
\hline $\mathrm{Se} 1$ & 1 & 1 & 1 & 1 \\
\hline Vitamines & - & 0,5 & - & - \\
\hline UF estimé & 0,72 & 0,64 & 0,74 & 0,72 \\
\hline MaD estimé & 77 & 80 & 80 & 75 \\
\hline
\end{tabular}

Ces diverses adaptations ainsi que les dates auxquelles elles ont été effectuées font l'objet du tableau $\mathrm{n}^{\circ} \mathbf{I}$.

\section{Introduction}

La graine de coton a déjà été largement utilisée en alimentation animale dans tous les pays producteurs de cette fibre. Son appétibilité, sa richesse en énergie et surtout en matières azotées en font un aliment de choix utilisé surtout, jusqu'à nos jours et dans les zones tropicales arides, pour supplémenter, en saison sèche, les animaux entretenus sur les pâturages naturels. (De nombreux travaux ont été publiés dans ce domaine par l'Université de Floride.)

Au Sénégal, la culture du coton, d'introduction récente, prend un développement important et on peut supposer que, dans l'avenir, une partie de la production de graine, utilisée pour l'heure et en totalité par l'huilerie, deviendra disponible pour lélevage.

L'embouche intensive, la production et l'exportation de viande apparaissent alors comme des moyens immédiats capables de valoriser ce produit. Or, ces techniques nouvelles qui rencontrent au Sénégal de nombreux facteurs favorables sont gênées dans leur extension par les disponibilités peu importantes des produits ou sous-produits capables de rentrer dans la composition de rations d'embouche.
Mise à la disposition des éleveurs, la graine de coton pourrait donc constituer un facteur important permettant un développement rapide des techniques de production intensive de viande.

C'est dans cette optique qu'a été réalisé l'essai d'embouche faisant l'objet de cette note.

Le premier objectif est donc d'étudier l'efficacité de la graine de coton non délintée introduite dans ce type de ration à base de coque d'arachide mélassée, mise au point au Laboratoire de l'Elevage de Dakar et déjà utilisée dans plusieurs essais antérieurs.

L'analyse bromatologique des rations II et III a donné les résultats présentés dans le tableau $\mathbf{n}^{\circ}$ II.

TABLEAU $\mathrm{N}^{\circ}$ II

Analyse bromatologique (p.1000 de M.S.)

\begin{tabular}{|l|c|c|}
\hline Matières sèches & Ration II & Ration III \\
Matières minêrales & 881,5 & 874,5 \\
Matières grasses & 81,5 & 71,8 \\
Matières azotées & 75,4 & 94,4 \\
(N x 6,25) & 140,6 & 141,8 \\
Cellulose (Wende) & 321,2 & 238,8 \\
Extractif non azotê & 381,3 & 453,2 \\
Phosphore & 8,86 & 4,59 \\
Calcium & 12,9 & 8,55 \\
\hline
\end{tabular}




\section{Contrôle de l'expérience}

- Les animaux sont pesés toutes lë quatre semaines, trois jours consécutifs. Le poids attribué est la moyenne des trois mesures (pesées de référence).

- La ration est pesée journellement de même que les refus.

- L'étude des carcasses concerne cinq animaux abattus en début d'expérience (témoins) et autant en fin d'essai. Les mesures et observations effectuées à l'abattoir suivent le protocole utilisé dans les expérimentations antérieures.

Comme au début de chaque essai, les animaux sont vaccinés contre la peste et la péripneumonie et soumis à un examen de coprologie en vue d'apprécier leur degré de parasitisme. Le service d'helminthologie conclut à une infestation faible; mais à titre d'expérience, la moitié de l'effectif des quatre lots est vermifugée au Tétramisole (*).

Une enquête concernant une éventuelle infestation des zébus par trypanosome donne des résultats négatifs.

\section{RESULTATS}

Les performances rapportées intéressent une période de 112 jours comprise entre le 26 mai et le 15 septembre, date à laquelle les animaux sont abattus.

La période antérieure allant du 30 avril au 26 mai est considérée comme «phase d'adaptation » ou de tâtonnements et cela pour deux raisons :

(*) Le produit utilisé est le 16.535 R.P tétramisole commercialisé sous le nom de Vadephen Specia.
- Les animaux achetés et tout partıculièrement les zébus Gobra et Maures étaient dans un très mauvais état général comme en témoignent les poids moyens en début d'essai, inférieurs à ceux enregistrés en général sur ce type d'animal. Les Ndama par contre, prélevés sur le troupeau de la ferme, étaient dans un bien meilleur état. Une période de rétablissement des zébus paraissait donc utile avant de pouvoir les comparer aux taurins.

- L'aliment distribué au cours de cette période d'adaptation contenait 27 p. 100 de graine de coton, ce qui s'est avéré excessif, compte tenu du taux élevé de gossypol contenu dans la graine (gossypol libre : 0,087 p. $100 \mathrm{du}$ produit brut) et du mauvais état des animaux qui recevaient cette ration. Le taux élevé de gossypol est certainement responsable du comportement des zébus et des Djakorés en ce début d'essai. En effet, le gain de poids est pratiquement nul et des amaigrissements aigus surviennent, qui contraignent à l'élimination de deux animaux. Pour parer aux effets de cette intoxication, le taux de graine de coton est diminué (18 p. 100) et le taux protéique de la ration augmenté. Le régime II témoigne de ces adaptations.

En ce qui concerne le déparasitage effectué sur la moitié de l'effectif, les performances obtenues chez les traités et non traités de chaque groupe ne sont pas significativement différentes (les valeurs de F chez les Maures, Gobra, Djakoré et Ndama sont respectivement de $1,29-0,48-1,30-0,50$ ). Le déparasitage n`a donc, dans le cadre de cette expérience, produit aucun effet sur les performances qui vont être analysées maintenant.

Le tableau $n^{\circ}$ III montre les poids moyens obtenus dans chaque lot, à chaque période et sur la totalité de l'essai.

TABLEAU $N *$ III

Evolution des poids moyens et gains de poids par pêriode

\begin{tabular}{|c|c|c|c|c|c|c|}
\hline $\begin{array}{c}\text { Nature } \\
\text { lot }\end{array}$ & $\begin{array}{l}\text { Nombre } \\
\text { animaux }\end{array}$ & $26 \mathrm{mai}$ & 23 juin & 21 juillet & 18 août & 15 septembre \\
\hline Maure & 9 & $222,9 \pm 18,4$ & $259 \pm 20,3$ & $293,6 \pm 16,7$ & $321,7 \pm 19,7$ & $351,9 \pm 21,1$ \\
\hline Gobra & 9 & $244,7 \pm 17$ & $278 \pm 19,4$ & $311,9 \pm 19$ & $336,6 \pm 19,9$ & $363,4 \pm 21,6$ \\
\hline Djakore & 9 & $235,8 \pm 24,2$ & $264,1 \pm 26,5$ & $291,5 \pm 29,2$ & $313,8 \pm 32,9$ & $340,9 \pm 33,3$ \\
\hline$N^{\prime}$ Dama & 10 & $224,4 \pm 28,8$ & $250,9 \pm 30,4$ & $269,9 \pm 30$ & $290,6 \pm 28,9$ & $\pm 29,4$ \\
\hline
\end{tabular}


TABLEAU $\mathrm{N}^{\bullet} \mathrm{TV}$

Gains de poíds observés

\begin{tabular}{|c|c|c|c|c|c|}
\hline & 28 jours & 28 jours & 28 jours & 28 jours & Totalitê \\
\hline & $26 / 5$ an $23 / 6$ & $23 / 6$ au $21 / 7$ & $21 / 7$ au $18 / 8$ & $18 / 8$ au $15 / 9$ & essai \\
\hline Maure & $36,1 \pm 5,7$ & $34,6 \pm 4,4$ & $28,1 \pm 5,3$ & $30,3 \pm 4,8$ & $129,0 \pm 12,2$ \\
\hline Gobra & $33,3 \pm 6,2$ & $33,9 \pm 3,3$ & $24,7 \pm 4,9$ & $26,7 \pm 6,3$ & $118,7 \pm 10,2$ \\
\hline Djakoré & $2 B, 3 \pm 6,3$ & $27,4 \pm 5,7$ & $22,3 \pm 6,4$ & $27 \pm 8,3$ & $105,1 \pm 16,2$ \\
\hline N'Dama & $26,5 \pm 7,9$ & $18,9 \pm 2,6$ & $20,7 \pm 4,4$ & $24,4 \pm 3,5$ & $90,6 \pm 10,4$ \\
\hline
\end{tabular}

TABLEAU $N^{\circ} \mathrm{V}$

Gains de poids journaliers (g)

\begin{tabular}{|l|c|c|c|c|c|}
\hline & 28 jours & 28 jours & 28 jours & 28 jours & $\begin{array}{c}\text { Totalité } \\
\text { easai }\end{array}$ \\
\cline { 2 - 6 } & $26 / 5$ au $23 / 6$ & $23 / 6$ au $21 / 7$ & $21 / 7$ au $18 / 8$ & $18 / 8$ au $15 / 9$ & 1.152 \\
\cline { 2 - 6 } Maure & 1.291 & 1.233 & 1.006 & 1.079 & 1.093 \\
Djakra & 1.187 & 1.211 & 863 & 955 & 929 \\
N'Dama & 1.012 & 978 & 796 & 965 & 809 \\
\hline
\end{tabular}

TABLEAU $N^{\circ} \mathrm{VI}$

Consommation par période (en $\mathrm{kg}$ )

\begin{tabular}{|l|c|c|c|c|}
\hline & lère période & 2e période & Je période & 4e période \\
\cline { 2 - 5 } Maure & $11,5 \pm 3,1$ & $11,2 \pm 1,3$ & 10,1 & 10,6 \\
Gobra & $11,9 \pm 2,8$ & $11,3 \pm 1,2$ & 10,4 & 10,8 \\
Djakoré & $10,8 \pm 2,3$ & $11,2 \pm 1,3$ & 10,1 & 10,4 \\
N'Dama & $10,2 \pm 2,3$ & $10,2 \pm 0,8$ & 9,9 & 10,2 \\
\hline
\end{tabular}

Le tableau $\mathrm{n}^{\circ} \mathrm{IV}$ indique les gains de poids correspondants, le tableau $\mathrm{n}^{\circ}$ VI les consommations observées.

L'analyse des résultats par période, une période correspondant à l'intervalle compris entre deux pesées de référence, autorise les remarques suivantes (tableau $\mathrm{n}^{\mathrm{0}} \mathrm{V}$ ):

Première période: Sa durée est de 28 jours $(26 / 5$ au 23/6). La ration distribuée comporte 18 p. 100 de graine de coton et la consommation, exprimée en $\mathrm{kg}$ de matière sèche par $100 \mathrm{~kg}$ de poids vif, est très élevée dans tous les lots :

4,2 pour les zébus maures;

4,0 pour les zébus Gobra;

3,8 pour les Djakorés;

4,2 pour les Ndama.
Les gains de poids moyens sont également d'un haut niveau puisqu'ils dépassent le $\mathrm{kg}$ dans la plupart des lots. Dans un ordre décroissant, ils s'établissent ainsi :

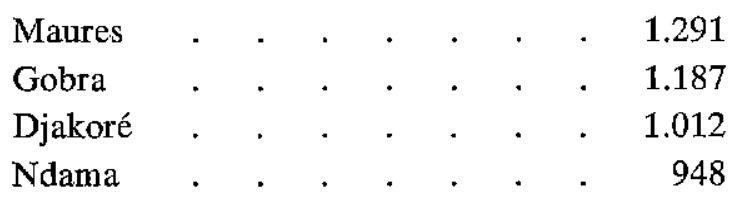

Les indices de consommation sont faibles et, dans un ordre croissant, se classent comme suit :

Maures . . . . . . . . 5,7

Gobra . . . . . . . . 6,4

Djakoré . . . . . . . 6,8

Ndama . . . . . . . . . 6,9

Deuxième période: Elle dure également 28 jours et s'étend du 23 juin au 21 juillet. 
Les troubles de la \& période d'adaptation * sont maintenant largement dépassés et on peut envisager de réaugmenter dans la ration la proportion de graine de coton, ce qui est fait au début de cette période. La ration passe alors de 18 à 24 p. 100 de graine de coton, ce qui équivaut à une valeur fourragère de 0,74 UF et 80 M.A.D.

D'autre part, devant la consommation excessive observée au cours de la $1^{\text {re }}$ période, et au début de celle-ci, l'aliment est rationné à $11 \mathrm{~kg}$ pour les zébus et les métis et $10 \mathrm{~kg}$ pour les Ndama à compter du 4 juillet.

La consommation en matière sèche par $100 \mathrm{~kg}$ de poids vif pour les quatre groupes et dans le même ordre est alors de : 3,5 - 3,3 3,5 - 3,3; ce qui correspond à des valeurs comparables à celles observées dans les essais antérieurs.

Les gains de poids journaliers et les indices de consommation au cours de cette période s'établissent ainsi :

$\begin{array}{llllrr}\text { Maures } & . & . & . & 1.233 \mathrm{~g} & 6,71 \mathrm{UF} \\ \text { Gobra } & . & . & . & 1.211 \mathrm{~g} & 6,91 \mathrm{UF} \\ \text { Djakoré } & . & . & . & 978 \mathrm{~g} & 8,47 \mathrm{UF} \\ \text { Ndama } & . & . & . & 676 \mathrm{~g} & 11,78 \mathrm{UF}\end{array}$

Les gains de poids diminuent chez les Ndama et les métis zébus Ndama. Les indices de consommation dans ces deux groupes augmentent en conséquence. Du point de vue de l'utilisation alimentaire, les Djakorés semblent se rapprocher davantage des Ndama que des zébus.

Troisième période: Sa durée est égale aux précédentes ( 28 jours). La formule de l'aliment et les quantités distribuées restent identiques, ce qui entraîne par rapport à la période précédente une diminution des quantités de matières sèches ingérées par $100 \mathrm{~kg}$ de poids vif $(2,9$ à $3,1 \mathrm{~kg}$ suivant les groupes).

On observe un léger fléchissement des performances qui peut être la conséquence du rationnement ou sous la dépendance des perturbations climatiques accompagnant le début de l'hivernage, qui se sont produites à ce moment là.

Les gains de poids et les indices de consommation sont les suivants :

Maures
Gobra

883 g 8,72 UF

Djakoré

$796 \mathrm{~g}$

9,39 UF

Ndama

$742 \mathrm{~g} \quad 9,91$ UF

Quatrième période : Elle s'étend du 18 août à la fin de l'essai le 15 septembre. Sa durée est identique aux précédentes ( 28 jours).

La composition de la ration est modifiée. La proportion de graine de coton reste identique, mais la farine de sorgho a dû être substituée à la farine de cône dont les stocks sont venus à épuisement.

La valeur de l'aliment est passée alors à 0,72 UF et 75 M.A.D. au kg.

Les conditions de rationnement restent identiques, ce qui se traduit encore par une légère diminution des quantités de matières sèches ingérées par $100 \mathrm{~kg}$ de poids vif $(2,7$ à $2,95 \mathrm{~kg})$.

En dépit d'une consommation moindre, les performances se rétablissent par rapport à la période précédente, ce qui laisse supposer une adaptation des animaux aux conditions de l'hivernage très peu marqué cette année.

Les gains journaliers et l'indice de consommation prennent les valeurs suivantes:

$\begin{array}{llllllll}\text { Maures } & . & . & . & . & 1.079 \mathrm{~g} & 7,05 \mathrm{UF} \\ \text { Gobra } & . & . & . & . & 955 \mathrm{~g} & 8,16 \mathrm{UF} \\ \text { Djakoré } & . & . & . & . & 965 \mathrm{~g} & 7,76 \mathrm{UF} \\ \text { Ndama } & . & . & . & . & 868 \mathrm{~g} & 8,43 \mathrm{UF}\end{array}$

\section{Performances sur l'ensemble de l'essai}

L'évolution des gains de poids et des indices de consommation font l'objet des tableaux VII et VIII et du graphique I.

Pour chacun des lots, les croîts quotidiens moyens et les indices de consommation moyens sur les 112 jours qu'a duré cet essai s'établissent ainsi :

$\begin{array}{lllllrll}\text { Maures } & \cdot & . & . & . & 1.152 \mathrm{~g} & 6,67 \mathrm{UF} \\ \text { Gobra } & \cdot & . & . & . & 1.093 \mathrm{~g} & 7,21 \mathrm{UF} \\ \text { Djakoré } & \cdot & . & . & . & 929 \mathrm{~g} & 8,04 \mathrm{UF} \\ \text { Ndama } & \cdot & . & . & . & 809 \mathrm{~g} & 8,88 \mathrm{UF}\end{array}$

Ces résultats classent la ration utilisée comme une des meilleures parmi celles qui ont été expérimentées jusqu'ici au Laboratoire de l'Elevage de Dakar. 
Retour au menu

TABLLAU $\mathbb{N}^{\circ} \mathrm{VII}$

Evolutıon gain moyen journalier par période et cumulé

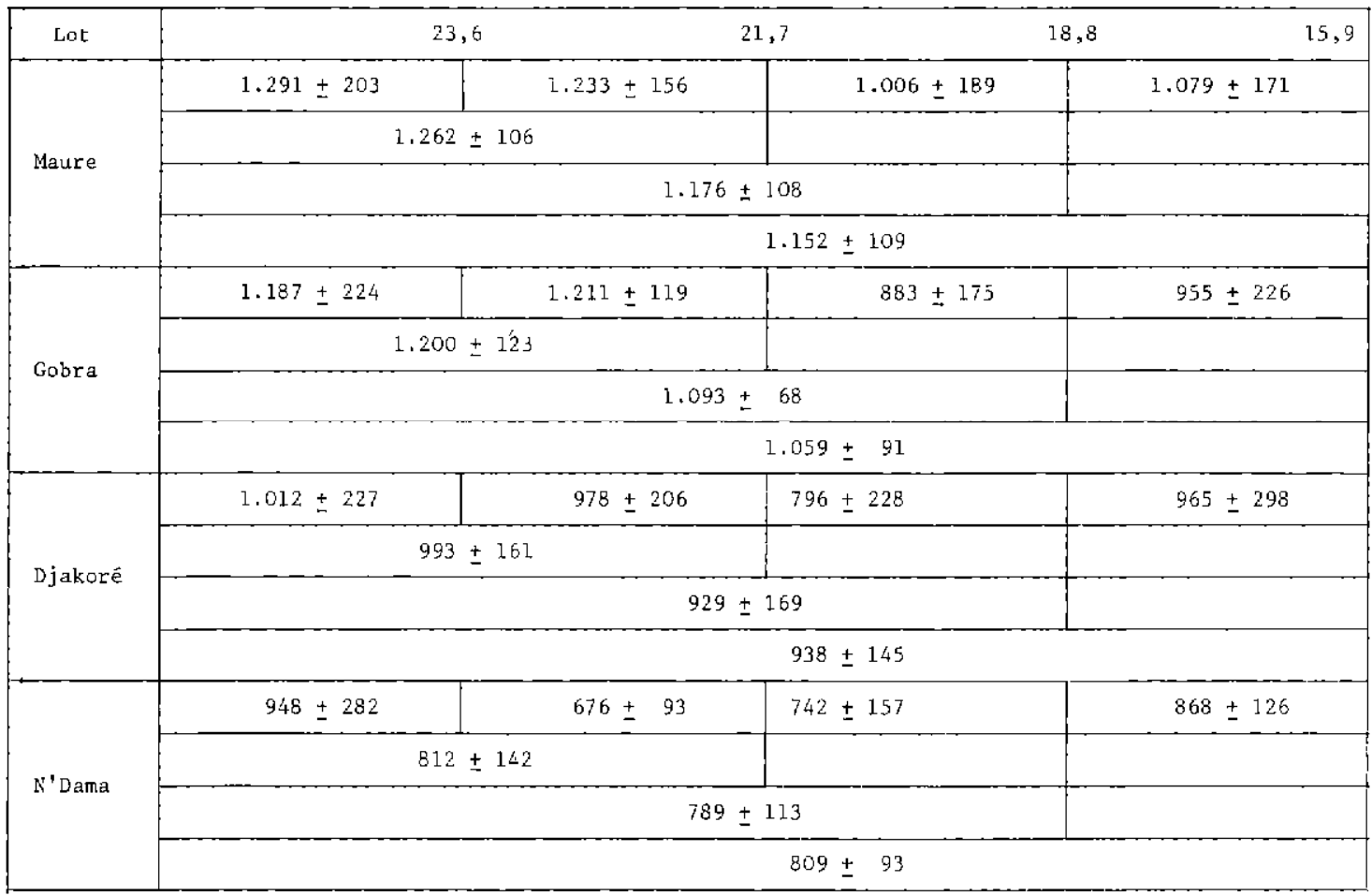

TABLEAU $\mathbb{L}^{\circ} \mathrm{VIII}$

Evolution de l'indice ce consommation par période et cumulé

\begin{tabular}{|c|c|c|c|c|}
\hline Lot & & & & 15,9 \\
\hline \multirow{4}{*}{ Maure } & 5,70 & 6,71 & 7,47 & 7,05 \\
\hline & \multicolumn{2}{|c|}{6,20} & & \\
\hline & \multicolumn{3}{|c|}{6,55} & \\
\hline & \multicolumn{4}{|c|}{6,67} \\
\hline \multirow{4}{*}{ Gobra } & 6,40 & 6,91 & 8,72 & $B, 16$ \\
\hline & \multicolumn{2}{|c|}{6,65} & & \\
\hline & \multicolumn{3}{|c|}{7,21} & \\
\hline & \multicolumn{4}{|c|}{7,41} \\
\hline \multirow{4}{*}{ Djakoré } & 6,84 & 8,47 & 9,39 & 7,76 \\
\hline & & & & \\
\hline & \multicolumn{3}{|c|}{8,14} & \\
\hline & & & 8,04 & \\
\hline \multirow{4}{*}{ iN'Dama } & 6,90 & 11,18 & 9,91 & 8,43 \\
\hline & & & & \\
\hline & \multicolumn{3}{|c|}{9,05} & \\
\hline & & & 8,88 & \\
\hline
\end{tabular}




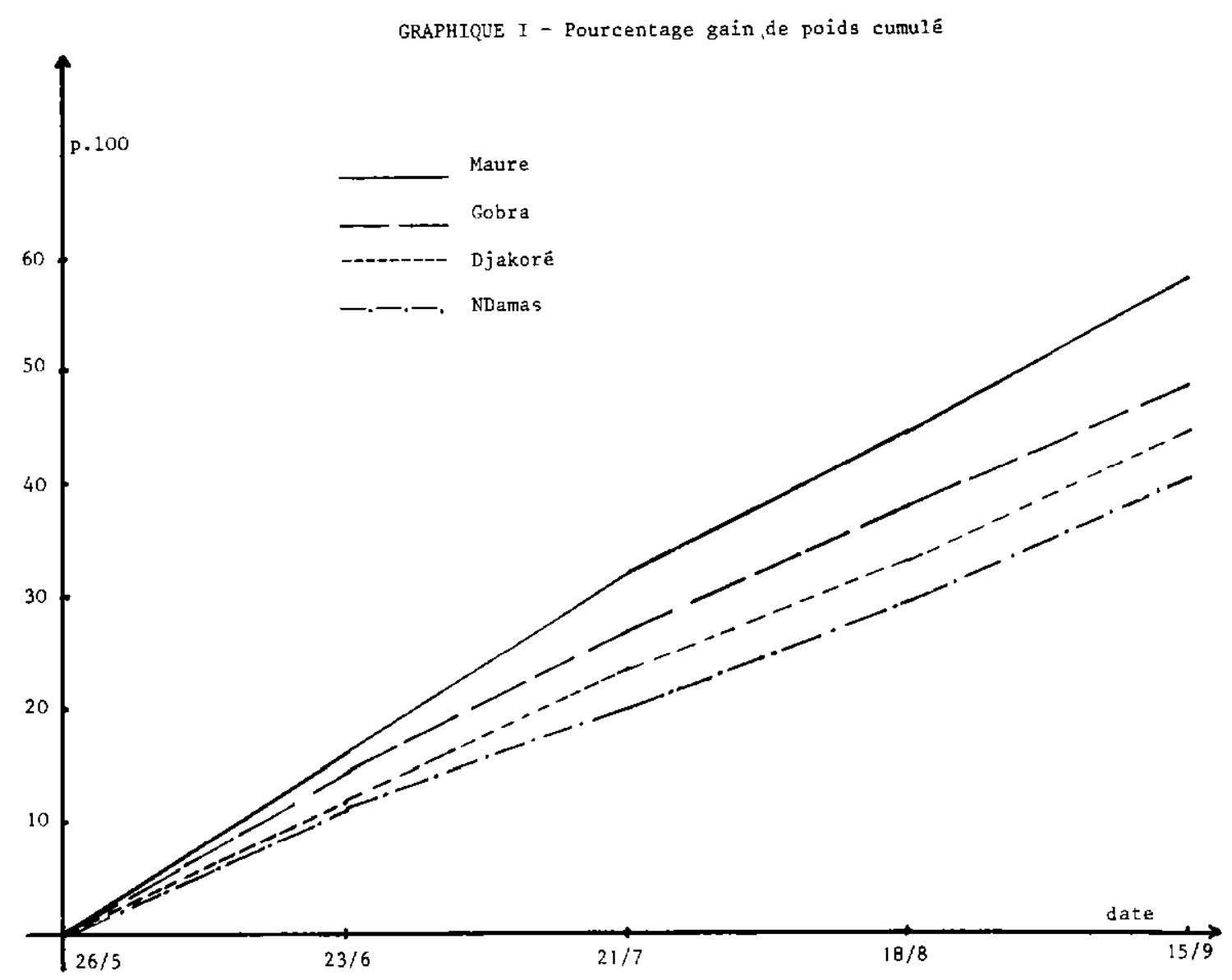

Les indices de consommation obtenus témoignent de l'efficacité de la ration mais aussi des bons effets du rationnement qui a évité les gaspillages de nourriture qui semblaient se produire dans la première période alors que la ration était distribuée ad libitum.

Enfin, en ce qui concerne les espèces et les races, les zébus Maures ont donné les meilleures performances, les Ndama les moins bonnes encore que le croît journalier moyen obtenu chez eux soit très honorable.

\section{Etude des carcasses}

Cinq animaux de chaque groupe sont abattus en début d'essai, mais trois carcasses sont saisies pour hydro cachexie et les abattages témoins ne portent plus que sur trois Maures, cinq Gobra, cinq Métis et quatre Ndama.

Les différences trop importantes existent sur les poids vifs, les poids carcasse et le rendement pour qu'on puisse établir une moyenne valable.

Cette première partie de l'étude est faite individuellement pour chaque animal abattu.
Les calculs ultérieurs, en particulier la proportion des différents morceaux, sont traités statistiquement avec la moyenne et l'intervalle de confiance à 5 p. 100. Les tableaux IX - X $\mathrm{XI}$ rapportent les différentes données obtenues au cours des abattages.

S'il est difficile de chiffrer l'augmentation du poids moyen de carcasses après quatre mois d'embouche en raison des différences individuelles, on peut toutefois l'estimer à 60 à $90 \mathrm{~kg}$ en moyenne selon les lots.

On constate surtout :

- une augmentation de 8 à 10 points des rendements et rendement vrai;

- une forte diminution du contenu de panse;

- une augmentation du pourcentage du globe sur tous les lots et de l'épaule sur tous, sauf pour les Gobra;

- une forte diminution du pourcentage du pis alors que celui du panneau ne varie pas;

- un état d'engraissement satisfaisant puisque l'indice de gras passe pratiquement de zéro à deux et plus. 
TABLEAU $N^{\circ} \mathrm{TK}$

Etude de carcasses

\begin{tabular}{|c|c|c|c|c|c|c|c|c|}
\hline \multirow[b]{4}{*}{ Maure } & \multirow[b]{3}{*}{ Témoins } & \multirow[t]{2}{*}{$\begin{array}{c}\text { Poids avant } \\
\text { jeûne }\end{array}$} & \multirow[t]{2}{*}{$\begin{array}{c}\text { Poids après } \\
\text { jeûne }\end{array}$} & \multicolumn{2}{|c|}{$\begin{array}{c}\text { Polds carcasse } \\
\text { chaude }\end{array}$} & \multicolumn{2}{|c|}{ Rendement } & \multirow[t]{2}{*}{$\begin{array}{c}\text { Poids carcasse } \\
\text { froide }\end{array}$} \\
\hline & & & & $\begin{array}{l}\text { Avec } \\
\text { collier }\end{array}$ & $\begin{array}{l}\text { Sans } \\
\text { collier }\end{array}$ & $\begin{array}{l}\text { Avec } \\
\text { collier }\end{array}$ & $\begin{array}{l}\text { Sans } \\
\text { collier }\end{array}$ & \\
\hline & & $\begin{array}{l}174 \\
207 \\
221\end{array}$ & $\begin{array}{l}164 \\
191 \\
209\end{array}$ & & $\begin{array}{l}73 \\
86 \\
90\end{array}$ & & $\begin{array}{l}44,5 \\
45,0 \\
43,1\end{array}$ & $\begin{array}{l}70 \\
83 \\
86\end{array}$ \\
\hline & $\begin{array}{l}\text { Fin } \\
\text { d'essai }\end{array}$ & $\begin{array}{l}337 \\
354 \\
341 \\
327 \\
429\end{array}$ & $\begin{array}{l}314 \\
333 \\
321 \\
314 \\
394\end{array}$ & $\begin{array}{l}178,7 \\
184,4 \\
178,2 \\
176,3 \\
227,4\end{array}$ & $\begin{array}{l}170 \\
178 \\
170 \\
169 \\
217\end{array}$ & $\begin{array}{l}56,9 \\
55,4 \\
55,5 \\
55,2 \\
57,7\end{array}$ & $\begin{array}{l}54,1 \\
53,5 \\
53,0 \\
53,8 \\
55,1\end{array}$ & $\begin{array}{l}168 \\
176 \\
169,3 \\
168 \\
214\end{array}$ \\
\hline \multirow{2}{*}{ Gobra } & Tẻmoins & $\begin{array}{l}250 \\
234 \\
208 \\
270 \\
205\end{array}$ & $\begin{array}{l}242 \\
222 \\
197 \\
254 \\
192\end{array}$ & & $\begin{array}{r}119 \\
101 \\
98 \\
109 \\
95\end{array}$ & & $\begin{array}{l}49,2 \\
45,5 \\
49,7 \\
42,9 \\
49,5\end{array}$ & $\begin{array}{c}116,3 \\
98,1 \\
94,4 \\
105,8 \\
92\end{array}$ \\
\hline & $\begin{array}{l}\text { Fin } \\
\text { d'essai }\end{array}$ & $\begin{array}{l}362 \\
410 \\
372 \\
315 \\
396\end{array}$ & $\begin{array}{l}344 \\
392 \\
355 \\
302 \\
374\end{array}$ & $\begin{array}{l}193 \\
238,5 \\
209 \\
176,8 \\
226,9\end{array}$ & $\begin{array}{l}184 \\
226 \\
199 \\
168 \\
215\end{array}$ & $\begin{array}{l}56,1 \\
60,8 \\
58,9 \\
58,5 \\
60,7\end{array}$ & $\begin{array}{l}53,5 \\
57,7 \\
56,1 \\
55,6 \\
57,5\end{array}$ & $\begin{array}{l}182 \\
222 \\
198 \\
165 \\
212\end{array}$ \\
\hline \multirow{2}{*}{ Djakorê } & Témoins & $\begin{array}{l}208 \\
212 \\
192 \\
216 \\
180\end{array}$ & $\begin{array}{l}193 \\
202 \\
176 \\
202 \\
180\end{array}$ & & $\begin{array}{l}88 \\
82 \\
81 \\
94 \\
75\end{array}$ & & $\begin{array}{l}45,6 \\
40,6 \\
46,0 \\
46,5 \\
41,7\end{array}$ & $\begin{array}{l}85 \\
77,5 \\
79 \\
91 \\
72\end{array}$ \\
\hline & $\begin{array}{l}\text { Fin } \\
\text { d'essai }\end{array}$ & $\begin{array}{l}339 \\
344 \\
354 \\
427 \\
332\end{array}$ & $\begin{array}{l}313 \\
320 \\
327 \\
400 \\
306\end{array}$ & $\begin{array}{l}182 \\
169,6 \\
195,3 \\
238,6 \\
184,4\end{array}$ & $\begin{array}{l}174 \\
163 \\
186 \\
228 \\
176\end{array}$ & $\begin{array}{l}58,1 \\
53,0 \\
59,7 \\
59,6 \\
60,3\end{array}$ & $\begin{array}{l}55,6 \\
50,9 \\
56,9 \\
57,0 \\
53,0\end{array}$ & $\begin{array}{l}172 \\
161 \\
183 \\
225 \\
173\end{array}$ \\
\hline \multirow[b]{2}{*}{ N'Dama } & Témoins & $\begin{array}{l}204 \\
229 \\
165 \\
142\end{array}$ & $\begin{array}{l}191 \\
214 \\
155 \\
133\end{array}$ & & $\begin{array}{l}93 \\
97 \\
69 \\
60\end{array}$ & & $\begin{array}{l}48,7 \\
45,3 \\
44,5 \\
45,1\end{array}$ & $\begin{array}{l}91 \\
95,5 \\
66 \\
56,5\end{array}$ \\
\hline & $\begin{array}{l}\text { Fin } \\
\text { d'essai }\end{array}$ & $\begin{array}{l}384 \\
268 \\
310 \\
271 \\
261\end{array}$ & $\begin{array}{l}361 \\
259 \\
287 \\
253 \\
239\end{array}$ & $\begin{array}{l}205,3 \\
139,6 \\
161,7 \\
142,2 \\
134\end{array}$ & $\begin{array}{l}197 \\
133 \\
153 \\
137 \\
129\end{array}$ & $\begin{array}{l}56,9 \\
53,9 \\
56,3 \\
56,2 \\
56,1\end{array}$ & $\begin{array}{l}54,6 \\
51,4 \\
53,3 \\
54,2 \\
54,0\end{array}$ & $\begin{array}{l}195 \\
131 \\
151,5 \\
136 \\
128\end{array}$ \\
\hline
\end{tabular}


Retour au menu

IABLEAC

Abatrages témoins

\begin{tabular}{|c|c|c|c|c|}
\hline & Maure & Gobra & Djakoré & $x$ Dama \\
\hline Pourcentage perte au jeûne & $6,68 \pm 1,4$ & $4,48 \pm 1,1$ & $6,68 \pm 3,2$ & $6,62 \pm 0,8$ \\
\hline Pourcentage perte au ressuyage & $3,54 \pm 0,9$ & $2,98 \pm 0,6$ & $3,71 \pm 1,4$ & $3,18 \pm 3,1$ \\
\hline Rendement sans collier & $44,20 \pm 2,4$ & $47,37 \pm 3,8$ & $44,8+3,4$ & $45,90 \pm 3,0$ \\
\hline Rendement vrai (sans collier) & $53,22 \pm 4,0$ & $53,56 \pm 2,2$ & $52,29 \pm 4,9$ & $53,30 \pm 2,5$ \\
\hline Pourcentage contenu panse & $16,84 \pm 9,7$ & $12,69 \pm 4,0$ & $16,39 \pm 5,2$ & $13,89 \pm 2,3$ \\
\hline Pourcentage 5e quartier & $32,17 \pm 5,8$ & $29,74 \pm 1,4$ & $31,48 \pm 4,4$ & $32,68 \pm 2,1$ \\
\hline En pourcentage de la carcasse & & & & \\
\hline . épau1e & $17,63 \pm 1,9$ & $22,90 \pm 0,2$ & $17,43 \pm 0,4$ & $15,69 \pm 2,9$ \\
\hline$\cdot \mathrm{p} 1 \mathrm{~s}$ & $18,61 \pm 2,0$ & $11,83 \pm 0,7$ & $19,23 \pm 1,9$ & $18,51 \pm 2,9$ \\
\hline - panneau & $4,91 \pm 1,4$ & $5,03 \pm 0,5$ & $4,68 \pm 0,6$ & $4,86 \pm 2,3$ \\
\hline - train de côtes & $10,45 \pm 4,6$ & $9,58 \pm 0,7$ & $10,94 \pm 1,4$ & $10,84 \pm 1,9$ \\
\hline globe & $47,16 \pm 2,7$ & $48,65 \pm 0,9$ & $46,08 \pm 1,9$ & $48,65 \pm 4,0$ \\
\hline bosse & 0,28 & $0,90 \pm 0,5$ & 0,32 & \\
\hline - gras de rugnon & 0,17 & $0,18 \pm 0,1$ & 0,21 & 0,20 \\
\hline - queue & $0,50 \pm 0,1$ & $0,60 \pm 0,1$ & $0,55 \pm 0,1$ & $0,60 \pm 0,2$ \\
\hline Longueur carcasse & $102,16 \pm 9,3$ & $106,1 \pm 6,1$ & $104,2 \pm 3,2$ & $98,87 \pm 9,5$ \\
\hline Epaisseur culsse & $15,43 \pm 6$ & $17,24 \pm 0,8$ & $15,3 \pm 1,3$ & $15,7 \pm 3,0$ \\
\hline Epaisseur plat de côtes & $1,86 \pm 0,2$ & $1,72 \pm 0,2$ & $1,78 \pm 0,2$ & $1,5 \pm 0,3$ \\
\hline
\end{tabular}

TABLEAU N ${ }^{\circ} \mathrm{XI}$

Abattage fin d'experience

\begin{tabular}{|c|c|c|c|c|}
\hline & Moure & Gobra & Djakoré & N'Dama \\
\hline Pourcentage perte du jeûne & $6,15 \pm 1,9$ & $4,72 \pm 0,7$ & $7,31 \pm 0,7$ & $9,80 \pm 3,9$ \\
\hline Pourcentige perte au ressuyage & $0,94 \pm 0,5$ & $1,30 \pm 0,7$ & $1,4 \pm 0,3$ & $1,0 \pm 0,4$ \\
\hline Rendement avec collier & $56,30 \pm 1,2$ & $58,96 \pm 2,4$ & $58,12 \pm 3,7$ & $55,82 \pm 1,4$ \\
\hline Rendement sans collier & $53,9 \pm 1,0$ & $56,08 \pm 2,1$ & $54,68 \pm 3,3$ & $53,5 \pm 1,6$ \\
\hline Rendenent vral avce collier & $60,92 \pm 0,6$ & $63,52 \pm 2,4$ & $61,78 \pm 3,8$ & $62,50 \pm 2,0$ \\
\hline Rondement vral suns collier & $38,33 \pm 0,8$ & $60,41 \pm 2,2$ & $59,08 \pm 3,4$ & $59,31 \pm 1,5$ \\
\hline l'ourcontage colliex & $\therefore 千+40,4$ & $2,94 \pm 0,3$ & $2,57 \pm 0,4$ & $2,41 \pm 0,5$ \\
\hline I'ourcentage contenu panse & $\overline{7},+\infty \pm \pm 2$ & $7,19 \pm 1,5$ & $5,92 \pm 0,5$ & $9,80 \pm 3,9$ \\
\hline $\begin{array}{l}\text { Pourcentale je quattier } \\
\text { in pourcentage de la careasse } \\
\text { iroide : }\end{array}$ & $28,50 \pm 3,4$ & $27,99 \pm 1,1$ & $29,13 \pm 2,2$ & $28,73 \pm 3,2$ \\
\hline · Eparals & $21,05 \pm 1,0$ & $20,98 \pm 1,7$ & $20,32 \pm 1,0$ & $22,08 \pm 2,1$ \\
\hline - pis & $12,18 \pm 1,1$ & $11,93 \pm 0,8$ & $12,30 \pm 0,6$ & $11,98 \pm 0,6$ \\
\hline$\cdot$ pinneiul & $\dot{4}, 13 \pm 0,7$ & $4,92 \pm 0,6$ & $4,50 \pm 0,4$ & $4,84 \pm 0,1$ \\
\hline - tran li sîle & $5,33 \pm 1.1$ & $8,64 \pm 0,8$ & $8,03 \pm 1,3$ & $8,51 \pm 1,0$ \\
\hline - qloje & $48,74 \pm 1,1$ & $48,47 \pm 2,2$ & $50,03 \pm 1,6$ & $49,27 \pm \therefore$, \\
\hline - bosse & $1,87 \pm 0,7$ & $2,22 \pm 0,6$ & $1,43 \pm 0,6$ & \\
\hline - quene & $0,6,4 \pm 0,1$ & $0,57 \pm 0,1$ & $0,67 \pm 0,1$ & $0,67 \pm 0,1$ \\
\hline - ras de rognon & $2,36 \pm 0,6$ & $2,07 \pm 0,8$ & $2 ; 30 \pm 0,6$ & $1,95 \pm 0,4$ \\
\hline lonrueur carcasse & $113,2 \pm 6,8$ & $112.2 \pm 3$ & $110,6 \pm 5,4$ & $103,7 \pm 8,6$ \\
\hline Arrisseur cusboe & $21,24 \pm 1,2$ & $23, .4 \pm 1,2$ & $23,26 \pm 1,5$ & $21,06 \pm 0,3$ \\
\hline 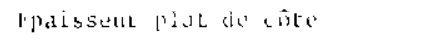 & $3,04 \pm 0,2$ & $3,38 \pm 0,2$ & $3,3 \pm 0,3$ & $3,14 \pm 0$ \\
\hline Indted de aras & $\therefore, 36 \pm 0,6$ & $2,07 \pm 0,8$ & $2,30 \pm 0,6$ & $1,95 \pm 0,4$ \\
\hline
\end{tabular}


TABLEAU $\mathrm{N}^{\circ} \mathrm{XII}$

Poids carcusses et des tiffeêrents morceaux ae gros avant et après embouche.

\begin{tabular}{|c|c|c|c|c|c|c|c|c|c|c|c|c|}
\hline & \multicolumn{2}{|c|}{ Maure } & \multirow{2}{*}{$\begin{array}{l}\text { P. } 100 \\
\text { en plus }\end{array}$} & \multicolumn{2}{|c|}{ Gobra } & \multirow{2}{*}{$\begin{array}{l}\text { P. } 100 \\
\text { en plus }\end{array}$} & \multicolumn{2}{|c|}{ Djakoré } & \multirow{2}{*}{$\begin{array}{l}\text { P. } 100 \\
\text { en plus }\end{array}$} & \multicolumn{2}{|c|}{ N'Dama } & \multirow{2}{*}{$\begin{array}{l}\text { P. } 100 \\
\text { en plus }\end{array}$} \\
\hline & Avanl & Après & & Avant & Áprès & & Avant & Aptès & & Avant & Après & \\
\hline $\begin{array}{l}\text { Carcasse } \\
\text { clraude }\end{array}$ & 92,3 & 1.78 & 82,0 & 110,7 & 194 & 75,24 & 97 & 173 & 79,3 & 96,2 & 153 & 59,0 \\
\hline Carcasse & 89 & 176,3 & 98,1 & 107,4 & 191,5 & 78,3 & 93,4 & 170,6 & 82,0 & 93,1 & 151,5 & 62,7 \\
\hline Epaule & 15,7 & 37,1 & 136,3 & 24.6 & 40,2 & 63,4 & 16,3 & 34,7 & 112,8 & 14,6 & 33,5 & 129,5 \\
\hline Pis & 16,6 & 21,5 & 29,5 & 12,7 & 22,8 & 79,5 & 18 & 21,0 & 16,7 & 17,2 & 18,1 & 5,23 \\
\hline Panncau & 4,4 & 7,3 & 65,9 & 5,4 & 9,4 & 74,1 & 4,4 & 7,7 & 75,0 & 4,5 & 7,3 & 62,2 \\
\hline $\begin{array}{l}\text { l'rain de } \\
\text { côtes }\end{array}$ & 9,3 & 14,9 & 60,2 & 10,3 & 15,5 & 60,2 & 10,2 & 13,7 & 34,3 & 10,1 & 12,9 & 27,7 \\
\hline Globe & 42,0 & 85,9 & $10_{4}^{\prime}, 5$ & 52,3 & 92,8 & 77,4 & 43 & 85,4 & 98,6 & 45,3 & 74,6 & 64,7 \\
\hline Posse & 0,25 & 3,3 & & 0,9 & 4,3 & & 0,3 & 2,4 & & & & \\
\hline $\begin{array}{l}\text { Gras de } \\
\text { rognon }\end{array}$ & 0,15 & 4,2 & & 0,2 & 4.0 & & 0,2 & 3,9 & & 0,2 & 3,0 & \\
\hline Queue & 0,4 & 1,1 & & 0,6 & 1.1 & & 0,5 & 1,1 & & 0,6 & 1,0 & \\
\hline
\end{tabular}

Arriōre = glube + Lran de sôte. Averl = épaule + prs + pannedu.

L'embouche entraîne donc non seulement une augmentation de la production de viande, mais aussi une très nette amélioration de la qualité (tableau $\pi^{\circ}$ XII). On peut estimer que le poids des carcasses mises à la disposition des professionnels de la viande est augmenté de 63 à 100 p. 100 et celui des morceaux nobles (train de côte + globe) de 60 à 96 p. 100 selon les races.

L'amélioration de la qualité est attestée par celle du rendement et de l'indice de gras.

\section{DISCUSSION}

Trois points vont être abordés successivement :

- la réactivité vis-à-vis du même aliment des différents types d'animaux mis en expérience;

-- l'influence des modifications alimentaires sur les performances dans chaque lot;

- l'esquisse économique de cette opération d'embouche.

\section{Comparaison des performances}

Elle est effectuée par analyse de variance des gains de poids individuels par période et pour toute la durée de l'essai.

En fonction des races:
Les gains moyens pour toute la durée de l'expérience classés par ordre décroissant sont les suivants:

- Maure $=129 \pm 12,2$

- Gobra $=118,7 \pm 10,2$

- Djakoré $=105,1 \pm 16,2$

- Ndama $=90,6 \pm 10,4$

L'analyse statistique montre qu'il y a entre les lots et d'une façon générale, une différence hautement significative $F=9,79$.

Cette différence existe pratiquement tout le long de l'expérience avec un degré de signification plus élevé lors de la deuxième période (tableau $\mathrm{n}^{\mathrm{v}}$ XIII).

L'analyse de variance montre que finalement, parmi les animaux étudiés, deux groupes se dessinent. D'une part, les zébus aux performances supérieures et d'autre part, les taurins et les métis Djakoré qui semblent se rapprocher davantage des taurins que des zébus.

En ce qui concerne le groupe zébu, les Maures et les Gobras n'ont pas présenté dans cet essai des performances significativement différentes, cependant celles des premiers ont tendance à être supérieures.

En fonction des périodes:

La comparaison des gains au cours de chaque période et dans chaque lot ne révèle à 
TABLEAU N*XIII

Comparaison entre les races - Valeurs de $F$

\begin{tabular}{|c|c|c|c|c|c|}
\hline & lère période & 2e période & 3e pĕriode & Le période & Total essai \\
\hline Maure/Gobra & 0,62 & 0,07 & 1,21 & 1,02 & 2,27 \\
\hline Maure/Djakor $\vec{e}$ & $4,49^{+}$ & $5,17^{+}$ & 2,66 & 0,58 & $7,43^{+}$ \\
\hline Maure/N'Dama & $4,82^{+}$ & $52,01^{++}$ & $6,10^{+}$ & $5,32^{+}$ & $30,31^{++}$ \\
\hline Gobra/Djakoré & 1,62 & $5,08^{+}$ & 0,48 & $0, \infty 0$ & 2,66 \\
\hline Gobra/N Dama & 2,21 & $67,61^{++}$ & 1,88 & 0,62 & $19,06^{++}$ \\
\hline Djakorê/N'Dama & 0,25 & $10,19^{++}$ & 0,20 & 0,51 & 3,10 \\
\hline$\frac{\text { (Maure }+ \text { Gobra) }}{\text { (Djakore }+ \text { N }^{\top} \text { Dama) }}$ & $6,49^{+}$ & $31,22^{++}$ & $4,77^{+}$ & 1,28 & $21,72^{++}$ \\
\hline
\end{tabular}

+ F significatif ou seuil 5 p.100; ++ F significatif ou seuil 1 p.100.

aucun moment de différences significatives. Les divers changements de la formule alimentaire n'ont donc entraîné aucune modification de comportement des animaux.

\section{Esquisse économique}

Elle se limite à un bilan entre, d'une part les charges représentées par l'achat et la nourriture des animaux et d'autre part, les recettes correspondant à la commercialisation des produits après embouche.

Le prix des animaux sur pied ou en carcasse a été étudié, ces temps derniers, par un groupe de travail constitué par le Ministère du Développement Rural du Sénégal et présidé par le Directeur de l'Elevage, en vue de proposer dans le domaine des prix les mesures capables d'assurer à Dakar un ravitaillement normal en viande (en évitant toute spéculation dans ce domaine).

Ce groupe, qui fait intervenir les critères de qualité, propose les prix suivants:

\section{- bétail vif au foirail de Dakar.}

L'animal de $1^{\text {re }}$ qualité, ayant subi une préparation pour la boucherie et un rendement carcasse supérieur à 50 p. 100 , devrait être commercialisé au prix moyen de $110 \mathrm{~F}$ le kg.

L'animal tout venant, avec un rendement compris entre 45 et 50 p. 100 devrait l'être au prix de $70 \mathrm{~F}$ le $\mathrm{kg}$.

- Carcasses à la cheville.

- carcasse de $1^{\text {re }}$ qualité : les arrières à $275 \mathrm{~F}$ le $\mathrm{kg}$; les avants à $165 \mathrm{~F}$ le $\mathrm{kg}$; - carcasse tout venant: $195 \mathrm{~F} \mathrm{le} \mathrm{kg}$.
Il sera tenu compte de ces propositions pour établir les recettes dans l'esquisse économique.

Les charges représentées par la nourriture des animaux sont calculées en fonction du prix d'achat des différents éléments entrant dans la composition des rations. Il n'est pas tenu compte des frais de fabrication.

Le prix de revient brut de l'aliment II est de $8,25 \mathrm{~F}$ CFA le kg, celui de l'aliment III est de $7,8 \mathrm{~F}$ le $\mathrm{kg}$ et celui de l'aliment IV dans lequel la farine de sorgho remplace la farine de riz est beaucoup plus élevé : $10,75 \mathrm{~F}$ le kg.

Compte tenu de ces bases, on peut établir les bilans suivants :

Cas de la vente des animaux sur pieds.

TABLEAU $\mathrm{N}^{*} \mathrm{XIV}$

Bilan économique des animaux sur pieds

\begin{tabular}{|l|c|c|c|c|}
\hline Race & $\begin{array}{c}\text { Prix } \\
\text { d'achat }\end{array}$ & $\begin{array}{c}\text { Frais } \\
\text { alimentation }\end{array}$ & $\begin{array}{c}\text { Prix de } \\
\text { vente }\end{array}$ & Bilan \\
\hline Maure & 15.610 & 10.500 & 38.720 & +12.610 \\
Gobra & 17.150 & 10.700 & 40.040 & +12.190 \\
Djakorêe & 16.520 & 10.300 & 37.510 & +10.690 \\
N'Dama & 15.750 & 9.800 & 34.650 & +9.100 \\
\hline
\end{tabular}

Le bénéfice brut réalisé après quatre mois d'embouche est de 9.000 à $12.600 \mathrm{~F}$ par animal avec un avantage pour les zébus maures grâce à un croît journalier supérieur et un indice de consommation inférieur à ceux des autres lots.

Cas de la vente en carcasse à la cheville.

Il est intéressant de comparer et de chiffrer la plus value des carcasses des mêmes animaux après embouche en tenant compte du tait que l'augmentation des taxes d'abattage et d'entre- 
posage due à une carcasse plus lourde est compensée par une meilleure commercialisation du $5^{\text {e }}$ quartier lui-même plus lourd et de meilleure qualité.

Le tableau XV ci-dessous indique cette plusvalue.

Là encore, l'avantage est accordé au zébu maure, mais le bénéfice brut est inférieur à celui réalisé lors de la vente des mêmes animaux sur pieds.

Comparaison avec les essais antérieurs.

Cette comparaison est faite uniquement sur les résultats de l'embouche et le coût de production du $\mathrm{kg}$ de croît des taurillons zébus Gobra de 4 à 5 ans recevant des rations différentes (tableau XVI).

Les différentes rations utilisées sont rappelées ci-après.
$1969:$

- Essai 1 : coque d'arachide mélassée et concentré à base de maïs et sorgho;

- Essai 2 : coque d'arachide mélassée et concentré à base d'issues de rizerie.

1970:

- Essai 3 : coque d'arachide mélassée et concentré à base de farine basse de riz et de son de maïs;

- Essai 4: paille de riz et concentré à base de farine basse de riz et son de maïs et mélasse.

1971:

Tous les lots reçoivent de la paille de riz et un concentré de composition variable.

- Lot I : concentré à base de mélasse farine de riz et son de maïs mélangé à de la paille hachée;

TABLEAU $\mathrm{N}^{\circ} \mathrm{XV}$

Bilan économique "carcasses"

\begin{tabular}{|l|c|c|c|}
\hline \multicolumn{1}{|c|}{ Race } & $\begin{array}{c}\text { Vente carcasse } \\
\text { avant embouche }\end{array}$ & $\begin{array}{c}\text { Frais } \\
\text { d'alimentation }\end{array}$ & $\begin{array}{c}\text { Vente carcasse } \\
\text { après embouche }\end{array}$ \\
\hline Maure & 17.355 & 10.500 & 38.600 \\
Gobra & 20.945 & 10.700 & 42.000 \\
Djakore & 18.215 & 10.300 & 37.700 \\
N'Dama & 18.155 & 9.800 & 33.800 \\
\hline
\end{tabular}

TABLEAU N*XVI

Comparaison avec rẻsultats antẻrieurs

\begin{tabular}{|c|c|c|c|c|c|c|}
\hline & $\begin{array}{c}\text { Durée } \\
\text { embouche }\end{array}$ & $\begin{array}{l}\text { Poids moyen } \\
\text { dêbut essai }\end{array}$ & $\begin{array}{l}\text { Poids moyen } \\
\text { fin essai }\end{array}$ & $\begin{array}{l}\text { Croit moyen } \\
\text { journalier }\end{array}$ & $\begin{array}{c}\text { Indice de } \\
\text { consommation }\end{array}$ & $\begin{array}{l}\text { Coût de } \\
\text { production du } \\
\text { kg de croitt }\end{array}$ \\
\hline \multicolumn{7}{|l|}{ Zêbu Gobra } \\
\hline 1969 - Essai 1 & 122 & 246,5 & 375,3 & 1.080 & 6,2 & $150 \mathrm{~F}$ \\
\hline Essai 2 & 122 & 248 & 318 & 585 & 10,3 & 88 \\
\hline 1970 - Essai 3 & 147 & 244 & 369 & 850 & 7,4 & 74 \\
\hline Essai 4 & 126 & 257 & 341 & 672 & 9,1 & 92 \\
\hline 1971 - Lot 1 & 111 & 271,5 & 349 & 698 & 8,6 & 91 \\
\hline Lot III & 111 & 271,9 & 354 & 739 & 7,8 & 144 \\
\hline Lot IV & 111 & 271,7 & 346,4 & 672 & 9,1 & 92 \\
\hline Lot V & 111 & 270 & 314,4 & 400 & 9,7 & 125 \\
\hline Lot VI & 111 & 284,6 & 331,6 & 423 & 8,3 & 93 \\
\hline 1972 & 112 & 245 & 364 & 1.059 & 7,4 & 90 \\
\hline 2ébu Maure & & & & & & \\
\hline 1972 & 112 & 223 & 352 & 1.152 & 6,7 & 82 \\
\hline
\end{tabular}


- Lot III : sorgho et son de blé remplacent la farine de riz et le son de maïs;

- Lot IV : même concentré que le lot $\mathrm{I}$, mais la paille n'est pas broyée;

- Lot V: tourteau d'arachide «expeller» uniquement;

— Lot VI : même tourteau plus urée alimentaire.

\section{2 :}

Coque d'arachide mélassée et concentré à base de graine de coton et farine basse de riz.

Les meilleurs résultats des points de vue croît journalier et indice de consommation chez les Gobras sont obtenus lors de l'essai $n^{\circ} 1 /$ 1969 avec l'aliment coque mélassée et concentré à base de maïs et sorgho, puis des essais 1972 et $3 / 1970$ où le concentré est à base de farine basse de riz; l'aliment paille de riz donne les moins bons résultats.

Du point de vue coût de la production du $\mathrm{kg}$ de croît, les meilleurs sont les aliments coque mélassée ou paille de riz et concentré à base de farine basse de riz, alors que ceux dont le concentré est à base de sorgho et de maïs entraînent les coûts de production les plus élevés.

Du point de vue général, performance et coût, ceux associant la coque mélassée, la farine de riz, le son de maïs avec ou sans graine de coton sont les meilleurs.

Mais c'est encore le zébu Maure qui valorise le mieux cet aliment.

\section{CONCLUSION}

La graine de coton, sous-produit déjà bien connu en alimentation animale, introduite dans des rations d'embouche à base de coque d'ara- chide mélassée, a permis d'obtenir au cours de cet essai de bonnes performances tant au point de vue des gains de poids et des indices de consommation, que des résultats économiques. Il faut signaler cependant que les quantités de graines à distribuer journellement aux animaux, doivent rester dans des limites assez précises en raison du gossypol contenu à des taux variables dans la graine.

Il est, en effet, apparu au début de cette expérimentation que le taux de 27 p. 100 de graine dans la ration, ce qui correspondait à une consommation de plus de $3,5 \mathrm{~kg}$ de graine par animal, n'était pas sans danger, compte tenu de la teneur en gossypol relativement élevée et de l'état très déficient des animaux mis en expérience. Il faut donc retenir de cet essai que l'utilisation de la graine de coton en alimentation animale et tout particulièrement quand il s'agit des rations d'embouche doit obéir à certaines règles qu'il conviendrait de préciser en fonction de la teneur en alcaloïde toxique. D'ores et déjà, on peut dire qu'il faut adapter progressivement les animaux à des quantités croissantes et que la distribution journalière ne devrait pas dépasser 2,5 à $3 \mathrm{~kg}$ de produit par animal et par jour.

L'autre problème évoqué dans cet essai, et qui était la réactivité comparée des zébus, des taurins Ndama et des métis des deux espèces, face à ce type de ration, a permis de mettre en évidence la supériorité des zébus sur les taurins, un léger bénéfice semblant se dessiner chez les Maures par rapport au Gobra. Les métis Djakoré, du point de vue des performances paraissent se rapprocher davantage des taurins que des zébus.

Enfin, le traitement anthelminthique effectué sur des animaux sahéliens présentant en saison sèche un faible taux d'infestation parasitaire n'a produit aucun effet sur les performances ultérieures.

\section{SUMMARY}

Cotton seed in intensive fattening.

\section{Comparative yields of cattle and zebu cattle and their metis}

The experiment carried in 1972 and reported in this paper was aimed in order to test and compare results of intensive fattening of four types living in Senegal; Gobra and Maure zebu cattle, Ndama and natural cross bred Gobra $\times$ Ndama, known as Djakore.

The experimental animals consisted in four or five years old bulls, weighting from 220 to $245 \mathrm{~kg}$. 
The ration fed was composed of a mixture of groundnut shells, molasses, cotton seed, rice meal and mineral, fed ad libitum during six weeks and then rationned in the amount of 10 to $11 \mathrm{~kg}$.

The results are following:

- Daily gains : from 809 to $1.152 \mathrm{~g}$,

- Energy conversion index from 6,6 to $8,8 \mathrm{UF} / \mathrm{kg}$

zebu cattle and specially Maure zebu cattle gained more than Ndama or Djakore.

This including cotton seed ration gave better results than others feeds used in the four experiments still reported.

\section{RESUMEN}

EI grano de algodón en el engorde intensivo.

Rendimientos comparados de Bos indicus, de Bos taurus y de sus mestizos

La experiencia notada en este artículo tiene por objeto de comprobar y de comparar los rendimientos obtenidos con un engorde intensivo de cuatro razas de bovinos: cebú Maure y Gobra; N'Dama y mestizo natural Djakoré encontrados en Senegal. Se distribuye ad libitum durante seis semanas, pues se raciona, el alimento constituido por cáscara de cacahuete con melaza, grano de algodón y harina de arroz. Los animales son novillos de 4 a 5 años de edad, de un peso medio de 200 a $245 \mathrm{~kg}$. Los resultados son los siguientes:

- incremento medio diario de peso de 809 a 1152 g según los lotes;

- indice medio de consumo de 8,88 a 6,67 .

Ponen en evidencia que los cebues, particularmente los Moros, tienen un mejor incremento que los N'Dama o Djakore.

La ración con grano de algodón es rentable sobretodo en los cebues y da mejores resultados que otros tipos de alimentación desde el punto de vista del rendimiento y del costo de producción.

\section{BIBLIOGRAPHIE}

1. CALVET (H.), VALENZA (J.), ORUE (J.), CHAMBON (J.) et WANE (A. M.). Engraissement intensif de zébus Peulhs sénégalais (Gobra) IV. Embouche en région rizicole - Mâles entiers ou castrés - poids moyen $250 \mathrm{~kg}$. Rev. Elev. Méd. vét. Pays trop., 1972, 25 (1) : 85-96.

2. VALENZA (J.), CALVET (H.), ORUE (J.) et WANE (A. M.). Engraissement intensif de zébus Peulhs sénégalais (Gobra). I. Mâles entiers 3 à 5 ans, Rev. Elev. Méd. vét. Pays trop., 1971, 24 (1) : $79-109$.
3. VALENZA (J.), CALVET (H.), ORUE (J.) et WANE (A. M.). Engraissement intensif de zébus peulhs sénégalais (Gobra). II. Mâles castrés 7-10 ans. Rev. Elev. Méd. vét. Pays trop., 1971, 24 (1) : 111-124.

4. VALENZA (J.), CALVET (H.), ORUE (J.) et WANE (A.M.). Engraissement intensif de zébus Peulhs sénégalais (Gobra). III. Mâles entiers ou castrés 3 à 5 ans et bœufs 7 à 9 ans. Rev. Elev. Méd. vét. Pays trop., 1971, 24 (4) : 597-634. 